\title{
FIES fiber injection upgrade
}

Stürmer, Julian; Seifahrt, Andreas ; Schwab, Christian ; Gandolfi, Davide ; Montanés Rodriguez, Pilar ; Buchhave, Lars

\section{Published in:}

Proceedings of SPIE 10702 - Ground-based and Airborne Instrumentation for Astronomy VII

Link to article, DOI:

$10.1117 / 12.2313052$

Publication date:

2018

Document Version

Publisher's PDF, also known as Version of record

Link back to DTU Orbit

Citation (APA):

Stürmer, J., Seifahrt, A., Schwab, C., Gandolfi, D., Montanés Rodriguez, P., \& Buchhave, L. (2018). FIES fiber injection upgrade. In C. J. Evans, L. Simard, \& H. Takami (Eds.), Proceedings of SPIE 10702 - Ground-based and Airborne Instrumentation for Astronomy VII (Vol. 10702). [107022S] SPIE - International Society for Optical Engineering. https://doi.org/10.1117/12.2313052

\section{General rights}

Copyright and moral rights for the publications made accessible in the public portal are retained by the authors and/or other copyright owners and it is a condition of accessing publications that users recognise and abide by the legal requirements associated with these rights.

- Users may download and print one copy of any publication from the public portal for the purpose of private study or research.

- You may not further distribute the material or use it for any profit-making activity or commercial gain

- You may freely distribute the URL identifying the publication in the public portal 


\section{FIES fiber injection upgrade}

Julian Stürmer, Andreas Seifahrt, Christian Schwab, Davide Gandolfi, Pilar Montañes-Rodriguez, et al.

Julian Stürmer, Andreas Seifahrt, Christian Schwab, Davide Gandolfi, Pilar Montañes-Rodriguez, Lars Buchhave, "FIES fiber injection upgrade," Proc. SPIE 10702, Ground-based and Airborne Instrumentation for Astronomy VII, 107022S (6 July 2018); doi: 10.1117/12.2313052

Event: SPIE Astronomical Telescopes + Instrumentation, 2018, Austin, Texas, United States 


\title{
FIES fiber injection upgrade
}

\author{
Julian Stürmer ${ }^{a}$, Andreas Seifahrt ${ }^{a},{\text { Christian } \text { Schwab }^{b} \text {, NOT staff }}^{c}$, Davide Gandolfi ${ }^{e}$, Pilar \\ Montañés Rodriguez ${ }^{d}$, Lars Buchhave ${ }^{f}$ \\ ${ }^{a}$ University of Chicago, USA; ${ }^{b}$ Macquarie Univ., Australia; ${ }^{c}$ Nordic Optical Telescope, Spain; ${ }^{d}$ \\ Instituto de Astrofísica de Canarias, Spain; ${ }^{e}$ Universitá degli studi di Torino, Italy; ${ }^{f}$ DTU \\ Space, National Space Institute, Technical University of Denmark, Denmark
}

\begin{abstract}
We report on the upgrade of the fiber link of FIES, the high-resolution echelle spectrograph at the Nordic Optical Telescope (NOT). In order to improve the radial velocity (RV) stability of FIES, we replaced the circular fibers by octagonal and rectangular ones to utilize their superior scrambling performance. Two additional fibers for a planned polarimetry mode were added during the upgrade. The injection optics and the telescope front-end were also replaced. The first on-sky RV measurements indicate that the influence of guiding errors is greatly suppressed, and the overall RV precision of FIES has significantly improved.
\end{abstract}

Keywords: optical fibers, radial velocity, FIES, echelle, spectrograph

\section{INTRODUCTION}

FIES is a cross-dispersed, high-resolution echelle spectrograph at the $2.56 \mathrm{~m}$ Nordic Optical Telescope. The instrument offers three resolution modes $\mathrm{R}=67,000$ (high-res), $\mathrm{R}=46,000$ (med-res) and $\mathrm{R}=25,000$ (low-res). The wavelength range recorded by the CCD is $370 \mathrm{~nm}$ to $940 \mathrm{~nm}$. However, for wavelengths longer than $800 \mathrm{~nm}$, the order spacing in the high-res mode is becoming too small to cleanly extract spectra. In the med-res mode, the extended wavelength range is available. FIES is most commonly used to measure stellar abundances, to study stellar activity and seismology, and for exoplanetary research. In the past, FIES has reached a RV precision of about $8 \mathrm{~m} \mathrm{~s}^{-1}$ for RV standard stars over timescales of days to weeks. ${ }^{1}$ On-sky tests have shown that its RV stability was mainly limited by insufficient spatial scrambling. Optical fibers with non-circular cross sections have been shown to improve spatial scrambling significantly ${ }^{2-4}$ and are now the standard choice for newly built RV spectrographs. ${ }^{5-7}$ They have also successfully been retrofitted to older instruments to improve their RV stability. ${ }^{8,9}$ Here, we report on the fiber injection upgrade for FIES.

\section{TELESCOPE FRONT-END}

In the process of replacing the circular fibers, the telescope front-end was also replaced for two reasons. The old injection system for calibration light only supported two different light sources. Since FIES will be upgraded with an etalon calibration source in the near future, an additional port became necessary. The second reason is that the old front-end projected the telescope pupil onto the near-field of the fibers. Although this allows minimizing the number of optical surfaces in the optical system that converts the telescope f-ratio to the faster f-ratio needed to feed the fiber efficiently, it is not optimal with respect to scrambling. Guiding errors affect the stellar image shape and position more than the telescope pupil. Non-circular fibers only effectively scramble the fiber near-field. As long as no double-scrambler (i.e. optics that exchange near- and far-field) is used, it is therefore beneficial to map the stellar image onto the fiber, rather than the telescope pupil. An additional motivation to change the front-end optics was that the old fiber bundle fed the light in a relatively slow beam ( f/6) into the fibers. Since focal ratio degradation (FRD) is lower for faster beams, we decided to use smaller fibers and a f-ratio of $\mathrm{f} / 4.8$.

The fiber input head at the telescope end of the fibers consists of 4 fibers with individual telecentric optical systems that convert the f/11 telescope beam to f/4.8 (FN-systems). One FN-system consists of two custom

Further author information: (Send correspondence to JS)

E-mail: stuermer@uchicago.edu

Ground-based and Airborne Instrumentation for Astronomy VII, edited by Christopher J. Evans, Luc Simard, Hideki Takami, Proc. of SPIE Vol. 10702, 107022S · ( 2018 SPIE · CCC code: 0277-786X/18/\$18 · doi: 10.1117/12.2313052 


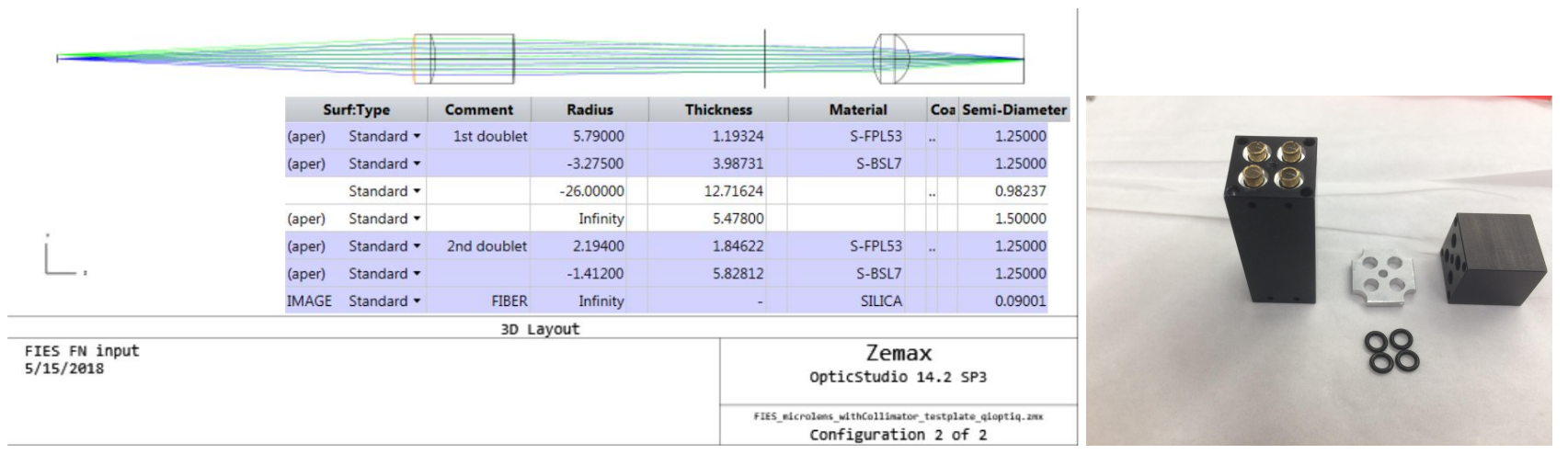

Figure 1: FIES FN-system design that convert the f/11 telescope beam to f/4.8 (left). All lenses have a diameter of $2.5 \mathrm{~mm}$ and are held in a thin brass tube. Due to optical constrains, all fibers had to sit closely (6 mm distance) together (right).

microlens doublets (manufactured by Qioptiq, Asslar, Germany) with $2.5 \mathrm{~mm}$ diameter, the same size as the fiber ferrule. The second microlens has a flat exit surface that is in physical contact with the fiber to minimize glass-air transitions. Lenses and fiber ferrule are both held in a single brass tube that is cut along its axis to provide radial clamping. The lens system provides diffraction limited performance over the entire wavelength range $(370 \mathrm{~nm}$ to $900 \mathrm{~nm}$ ) and the lenses are AR coated on all air-glass surfaces with an average of $99 \%$ transmission from $400 \mathrm{~nm}$ to $900 \mathrm{~nm}$. The FN design and the partially assembled fiber head is shown in Figure 1.

Placed in front of each FN-system is a $12.7 \mu \mathrm{m}$ thin metallic mirror with a precision machined central hole with either $210 \mu \mathrm{m}$ (med- and high-res) or $350 \mu \mathrm{m}$ (low-res) diameter, manufactured by National Aperture. The mirrors sit on a slanted surface and reflect the light of the PSF wings to a camera for guiding. Each mirror was individually aligned to optimize the registration of the hole with respect to the actual fiber position.

Each fiber can be illuminated by calibration light. The new front-end has four calibration fiber ports. Inside the front-end, one fiber from each port is merging into a single fiber connector. A subsequent FN-system and mirrors create a f/11 beam that can be moved in front of each fiber via a precision XY stage (see Fig. 2).

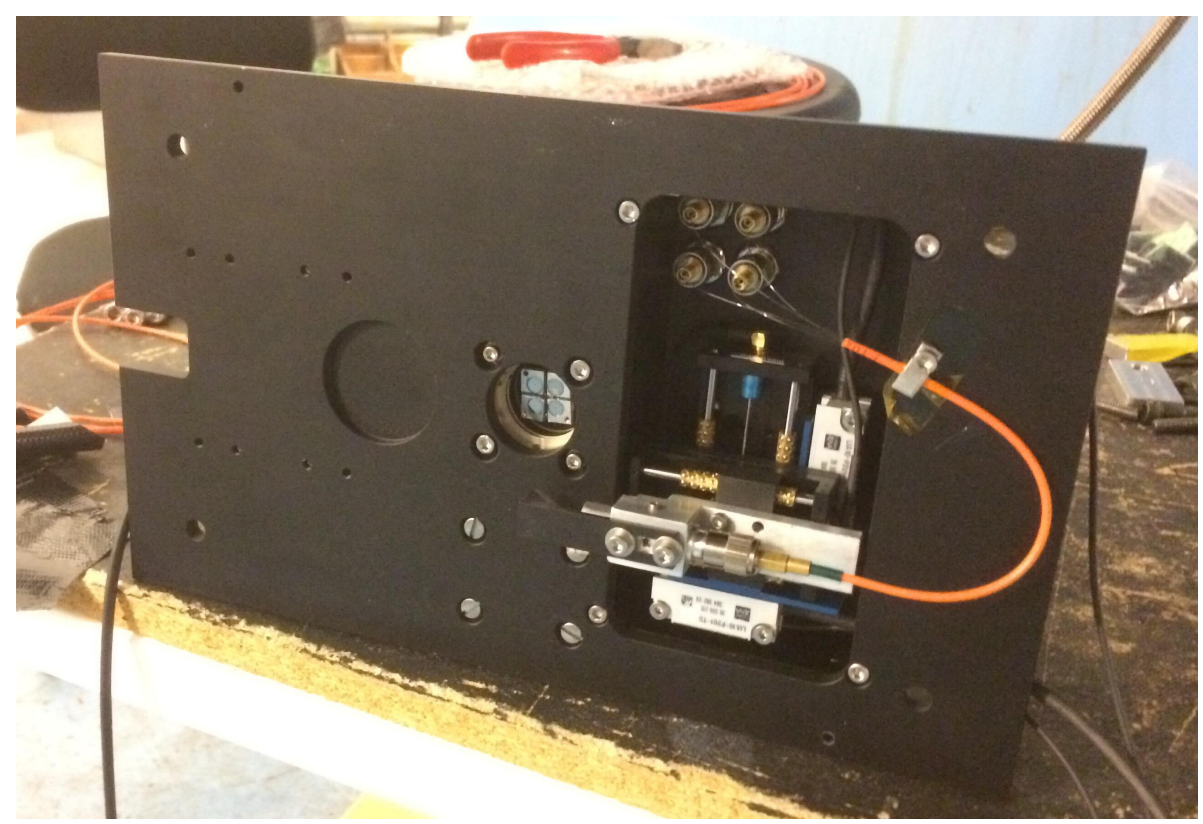

Figure 2: Part of the FIES telescope front-end. In the center, the four metallic guiding mirrors are visible. At the right sits the XY-stage that moves the calibration arm in front of the science fibers. 


\section{OPTICAL FIBERS}

In order to support all three resolution modes of FIES, three different fibers were used: Two octagonal (CeramOptec WF Oct 179/250A and CeramOptec WF Oct 89/125A) for the low- and med-res modes, and a 4:1 rectangular fiber (CeramOptec WF RCT 45x180/52x207/360/450A) for the high-res mode. Both octagonal fibers were drawn from the same preform. The total length of each fiber is $42 \mathrm{~m}$, intersected by FC-FC connectors after about $40 \mathrm{~m}$.

The $2 \mathrm{~m}$ fiber bundle at the spectrograph side ends with a fused silica slit plate (manufactured by FemtoPrint) with precision machined holes where the fibers are glued in (see Figure 3). In the old fiber bundle, the smaller slit width for the high-resolution mode was realized by masking part of the circular fiber. Now, the high-resolution mode consists of an octagonal fiber at the telescope end and a subsequent rectangular fiber, connected only by physical contact. Both methods lead to a slit loss of about $40 \%$. A planned image slicer should minimize this light loss and can easily be integrated as an upgrade at the fiber intersection (see section 5).

The smallest structural feedthrough of $10 \times 40 \mathrm{~mm}$ between the telescope and the spectrograph posed a tight mechanical constraint for the fiber routing and limited the size of the connectors and mechanics connected to the fibers. We therefore decided to divide the fibers into a long $40 \mathrm{~m}$ section and a short $2 \mathrm{~m}$ section, connected via FC-FC fiber connectors. The additional light loss due to the junction was measured in the lab to be $2 \%$ to $3 \%$. All fibers are protected by individual furcation tubes (Thorlabs FT020) inserted into a common stainless steel braided tube.

After installing the fiber bundle, simple tests using calibration light showed that the fibers suffered from focal ratio degradation, which led to an additional loss of about $25 \%$. Since previous tests in the lab did not show any issues, we suspect that fiber installation led to mechanical stress inside the bundle and therefore the increase in FRD. We hope to improve on the protection tube design and fiber routing when delivering the spare fiber bundle, which currently is still in the laboratory.

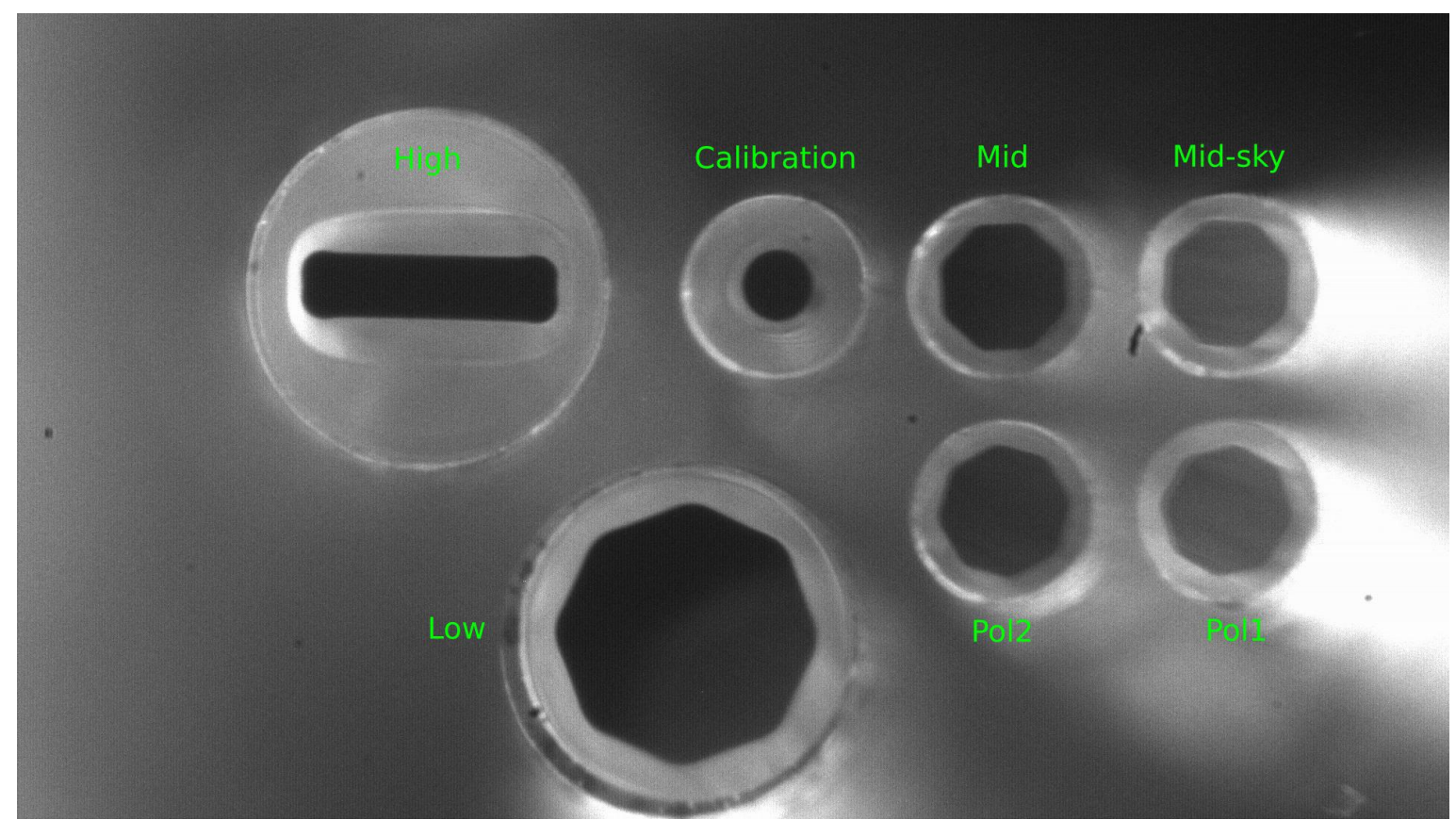

Figure 3: New FIES fiber exit slit plate illuminated from the side. The fibers are glued into a fused silica plate with precisely etched holes, and then polished. Clearly visible is the double cladding structure of the rectangular fiber (upper left). The dispersion direction is the y-direction. 


\section{ON SKY RESULTS}

In order to test the improvements on the scrambling gain from the octagonal fibers, we observed a RV standard star (HD 185144) and tested its RV stability against guiding offsets during commissioning. We took a series of 11 stellar spectra and intermediate ThAr calibration frames in the high resolution mode while offsetting the star in $0.25^{\prime \prime}$ steps for the last 4 spectra. The resulting RVs were unaffected by the spatial offset of the star, contrary to the results of previous tests with the old bundle, where offsetting the star led to an RV shift of over hundreds of meters per second (see Fig. 4). The RMS scatter (corrected for mostly pressure-induced shifts of the spectrograph via the ThAr frames) was measured to be $1.8 \mathrm{~m} \mathrm{~s}^{-1}$, a previously unattainable short-term stability.
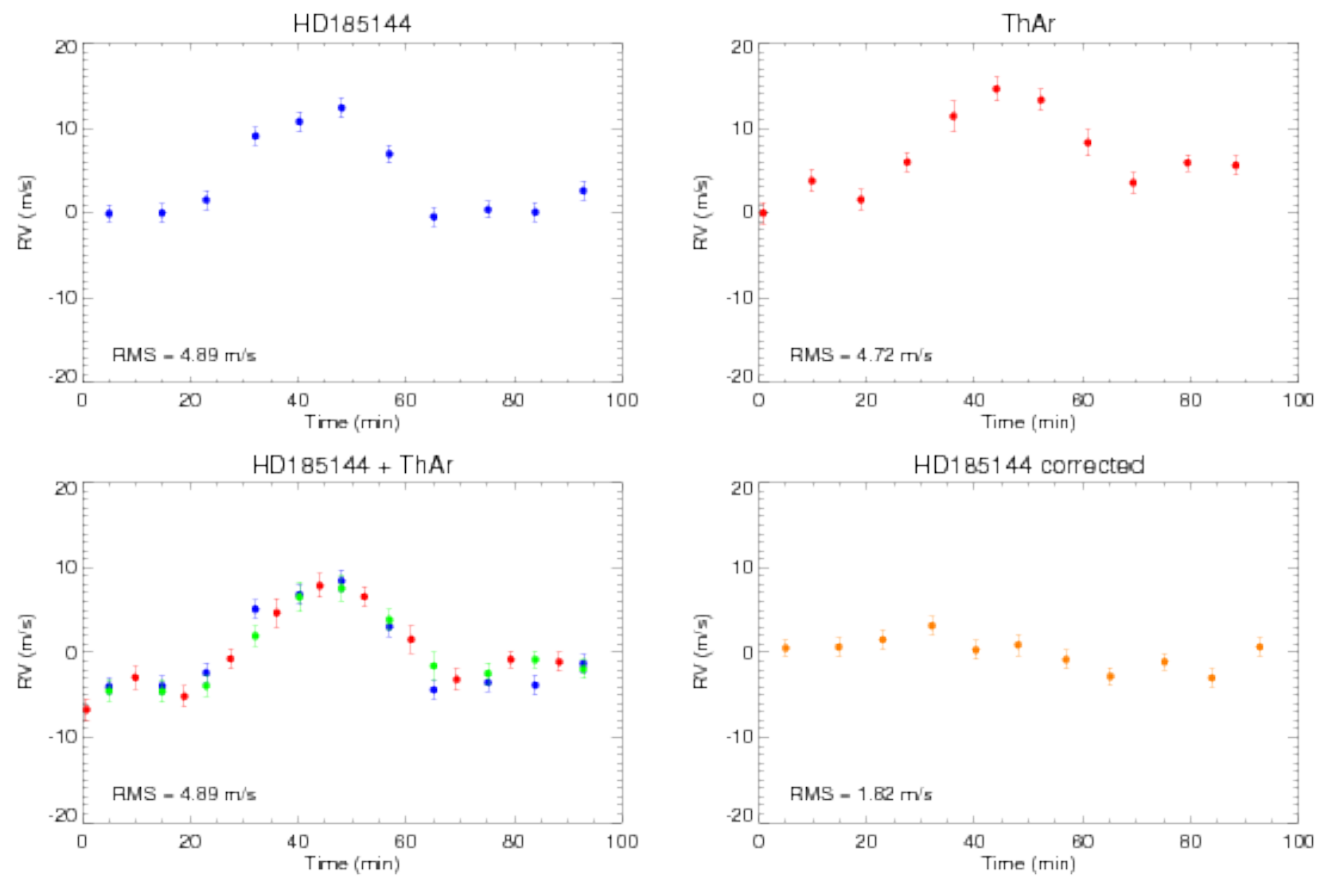

Figure 4: RV measurement of the RV standard HD185144 during commissioning of the new fiber bundle. For the last four spectra in this sequence, we deliberately offset the star on the guiding aperture in $0.25^{\prime \prime}$ steps to test the RV sensitivity to guiding errors. No significant change in RV was measured, demonstrating the greatly improved scrambling properties of the octagonal/rectangular fiber feed.

Another series of observations of an RV standard star using the medium resolution mode also showed excellent short term stability with an RMS scatter of $1.2 \mathrm{~m} \mathrm{~s}^{-1}$ (see Fig 5). On longer timescales, the achievable RV precision is currently under investigation, but we have strong indications that it also improved significantly.

\section{CONCLUSION AND OUTLOOK}

We reported on the successful upgrade of the fiber injection system of the FIES spectrograph at the NOT. By replacing the regular circular fibers with non-circular ones, we were able to improve the short term RV stability of FIES significantly to about $1 \mathrm{~m} \mathrm{~s}^{-1}$ to $2 \mathrm{~m} \mathrm{~s}^{-1}$. The increased RV precision allows targeting of a significantly larger set of exoplanet candidate systems, with smaller planetary companions. The upgrade of FIES is not fully completed, yet. While the spectrograph is mounted in a temperature controlled room, it is not in vacuum and consequently suffers from large RV drifts caused by the variations in ambient pressure. A precise wavelength solution and an exact determination of the spectrograph RV drift are thus crucial to obtain precision RV measurements. To address this, a stabilized and actively scanned Fabry-Perot etalon will be deployed in the coming year as an additional calibration source. ${ }^{10,11}$ Additionally, we are working on an image slicer that will improve the coupling efficiency between the octagonal fiber coming from the telescope and the 4:1 rectangular high-res fiber fed into the spectrograph. 


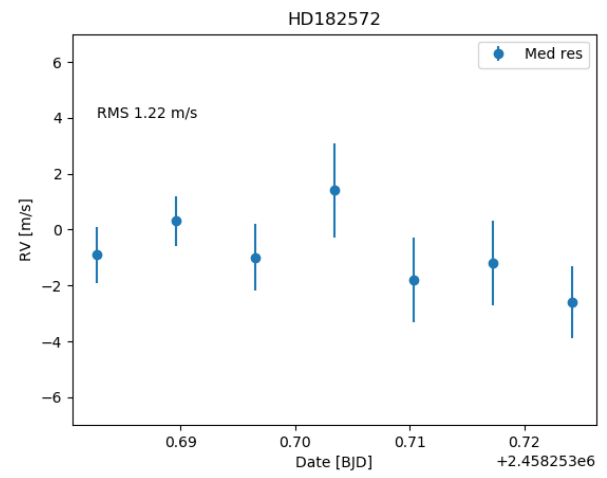

Figure 5: Another series of observations for a RV standard star, this time using the medium resolution mode, also shows excellent short term RV stability.

\section{REFERENCES}

1. J. Telting, G. Avila, L. Buchhave, S. Frandsen, D. Gandolfi, B. Lindberg, H. Stempels, the NOT staff, and S. Prins, "Fies: The highresolution fiberfed echelle spectrograph at the nordic optical telescope," Astronomische Nachrichten 335(1), pp. 41-45.

2. G. Avila and P. Singh, "Optical fiber scrambling and light pipes for high accuracy radial velocities measurements," in Advanced Optical and Mechanical Technologies in Telescopes and Instrumentation, Proc. SPIE 7018, p. 70184W, July 2008.

3. J. F. P. Spronck, D. A. Fischer, Z. A. Kaplan, C. Schwab, and A. Szymkowiak, "Fiber Scrambling for High-Resolution Spectrographs. I. Lick Observatory," PASP 125, p. 511, May 2013.

4. B. Chazelas, F. Pepe, F. Wildi, F. Bouchy, S. Perruchot, and G. Avila, "New scramblers for precision radial velocity: square and octagonal fibers," in Modern Technologies in Space- and Ground-based Telescopes and Instrumentation, Proc. SPIE 7739, p. 773947, July 2010.

5. J. Stürmer, O. Stahl, C. Schwab, W. Seifert, A. Quirrenbach, P. J. Amado, I. Ribas, R. A., and J. Caballero, "Carmenes in spie 2014. building a fibre link for carmenes," 2014.

6. S. Halverson, A. Roy, S. Mahadevan, L. Ramsey, E. Levi, C. Schwab, F. Hearty, and N. MacDonald, "An Efficient, Compact, and Versatile Fiber Double Scrambler for High Precision Radial Velocity Instruments," ApJ 806, p. 61, June 2015.

7. A. Roy, S. Halverson, S. Mahadevan, and L. W. Ramsey, "Scrambling and modal noise mitigation in the Habitable Zone Planet Finder fiber feed," in Ground-based and Airborne Instrumentation for Astronomy V, Proc. SPIE 9147, p. 91476B, July 2014.

8. F. Bouchy, R. F. Diaz, G. Hébrard, L. Arnold, I. Boisse, X. Delfosse, S. Perruchot, and a. Santerne, "SOPHIE+: First results of an octagonal-section fiber for high-precision radial velocity measurements," Astronomy E Astrophysics, p. 12, 2012.

9. G. Lo Curto, F. Pepe, G. Avila, H. Boffin, S. Bovay, B. Chazelas, A. Coffinet, M. Fleury, I. Hughes, C. Lovis, C. Maire, A. Manescau, L. Pasquini, S. Rihs, P. Sinclaire, and S. Udry, "HARPS Gets New Fibres After 12 Years of Operations," The Messenger 162, pp. 9-15, Dec. 2015.

10. C. Schwab, J. Stürmer, Y. V. Gurevich, T. Führer, S. K. Lamoreaux, T. Walther, and A. Quirrenbach, "Stabilizing a fabry perot etalon peak to $3 \mathrm{~cm} / \mathrm{s}$ for spectrograph calibration," Publications of the Astronomical Society of the Pacific 127(955), p. 880, 2015.

11. J. Stürmer, A. Seifahrt, C. Schwab, and J. L. Bean, "Rubidium-traced white-light etalon calibrator for radialvelocity measurements at the cm/s level," JATIS 3, pp. 3-3-10, 2017. 